\title{
NEXT GENERATION MOBILE COMMUNICATION SYSTEM WITH COGNITIVE RADIO, OFDM AND BEAMFORMING TECNIQUE
}

\author{
ARUN KUMAR ${ }^{1}$, HEMANT RATHORE ${ }^{2}$, SHIKHA BHARTI ${ }^{2}$ \\ ${ }^{1}$ Asst. Professor, Dept. of ECE JECRC UNIVERSITY, JAIPUR, INDIA, ${ }^{2}$ Dept. of ECE, JECRC UNIVERSITY, \\ JAIPUR, INDIA \\ E-mail: arun.kumar@jecrcu.edu.in, rathore1994hemant@gmail.com, shikhabharti712@gmail.com
}

\begin{abstract}
This work explores beam forming and two-way relaying to alleviate the dual issues of the spectrum congestion and spectrum under-utilization. These problems can be alleviated by the implementation of underlay cognitive radio networks. However, their reliability, capacity or power, and coverage depend on bi-directional or abbreviated as primaryto-secondary (P2S) and secondary-to-primary (S2P) interference constraints. This work makes of two-way relaying to improve these essential and paramount efficiencies, performance and effectiveness measures.
\end{abstract}

Keywords: Beam forming, MIMO, BER, Primary user $(\mathrm{Pu})$, Secondary user $(\mathrm{Su})$

\section{INTRODUCTION}

Radio transmissions have grown tremendously since the early development in the late nineteenth and the starting of twenty centuries and now influenced the living of users in every corner of the globe. As a precious resource, the radio spectrum must be carefully managed to mitigate spectrum pollution, maximize the utilization, and minimize the interference. In diversified and enormous countries, wireless systems or abbreviated as commercial or government operated have been allocated or abbreviated as licensed. To fulfil and cater the escalating requirements, new broadband transmission technologies have been introduced to utilize radio spectrum effectively [1]. This escalation motivates and higher the multi-user (Multiple Input and Multiple Output) MIMO network to achieve and get the similar capacity or power at the time the access-point having wireless antenna's transmission with the users [2]. MIMO means use multiple of antennas at transmitter and receiver. In addition to that, the more diversified gain is achieved when every user is having multiple antennas. Multi-user MIMO implementation is associated with IEEE-802.11ac or abbreviated as at the time of start 2014 to enable the multiple stations based WLAN having the throughput and efficiency of at least 1Gbps [3]. A very large MIMO system, known as massive MIMO that is, having arrays of largescale based antenna that is powerful and capable and powerful with a lessening of size of cell and reduction in the transmission power with the overhead for training channel [4]. In order to improve the effectiveness of the system, MIMO is combined with Orthogonal Frequency Division Multiplexing (OFDM). In OFDM. The single carrier is divided into number of subcarriers which are orthogonal to each other which in turns maximizes the throughput of the system $[5,6,7]$. The work discussed the power control and beam forming for the uplink of a secondary cognitive radio network that coexist with primary network. Giving the protection of primary user from harmful interference induced by the secondary user a priority, we have presented two iterative algorithms based on two different approach simulation result show that both algorithm are capable of keeping the interference level at the primary user below a specified threshold [8]. In this paper, a new relay based scheme for $\mathrm{CR}$ (cognitive radio) communication is proposed and analysed. By accurately designing the Beam forming (BF) weights at the relay node and taking into account the impact of imperfect CSI, we show that the proposal scheme outperforms the conventional scheme in term of secondary channel capacity and also circumvents the effect of imperfect CSI on the primary outage probability [9]. The work discussed the implementation of underlay cognitive radio network to enhance the reliability, capacity or power and coverage of the system. A two-way relaying technique is used for increasing the performance and effectiveness of the framework [10]. Adaptive beam-forming (AB) techniques are used for interference reduction by null broadening (NB) of the beam patterns of a cognitive radio base station in down-link operations. In this way, by steering beam pattern towards the selected regions the licensed users will experience a less interference [11]. The paper is structured as follows: Section I describes the introduction and similar work done. Section II presents the methodology, Section III described the results and discussion. Finally, section IV presents the conclusion of the work.

\section{PROPOSED METHODOLOGY MATHEMATICAL ANALYSIS}

MIMO-OFDM based System with 2 Transmitters and 2 receivers is used in this work with total enormous and diversified $\mathrm{N}$ subcarriers. $\mathrm{X}_{\mathrm{k}}^{\mathrm{t}}$ is modulated data using suitable modulation techniques for subcarrier. After modulation, the mapping of modulated data is done so that on the first and second antenna same data is transmitted and modulated OFDM symbols. Signal after Inverse fast Fourier transform (IFFT) at the transmitter of transmitting module can be written as [12].

$$
x^{t}(\mathrm{n})=\sum_{k=0}^{N-1} X_{k}^{t} e^{j\left(\frac{2 \pi}{N}\right) k n} \text { for } 0 \leq \mathrm{n} \leq \mathrm{N}-1
$$

$\mathrm{t}=(1 \text { or } 2, \mathrm{j}=\text { or abbreviated as }-1)^{1 / 2}$ is transmitter of transmitting module antenna. The received signal afflicted by phase noise and frequency offset can be expressed as [13]. 


$$
\begin{gathered}
r^{\tau}(n)=\left\{\sum _ { t = 1 } ^ { 2 } \left[x^{\prime}(n) * h^{\prime}(n)+\right.\right. \\
w(n)]\} e^{j\left[2 \pi \Delta f^{\tau} t+\varphi^{\tau}(n)\right]}
\end{gathered}
$$

$\tau=1$ or $2, \Delta \mathrm{f}^{\tau}$ and $\Phi^{\tau}(\mathrm{n})$ are frequency offset and phase noise. $\tau$ is received antenna. $\mathrm{x}(\mathrm{n}), \mathrm{h}(\mathrm{n}), \mathrm{w}(\mathrm{n}), \mathrm{r}(\mathrm{n})$ are transmitting signals, channel impulse response, Additive White Gaussian Noise (AWGN) and received signal respectively. The received signal after Fast Fourier transforms (FFT) can be written as [14].

$$
\begin{gathered}
Y_{k}^{\tau}=\frac{1}{N} \sum_{n=0}^{N-1} r^{\tau}(n) e^{-j\left[\frac{2 \pi}{N}\right] k n} \\
=\frac{1}{N} \sum_{n=0}^{N-1} \sum_{t=0}^{2} \sum_{l=0}^{N-1} X_{l}^{t} H_{l}^{t} e^{j\left[\left(\frac{2 \pi}{n}\right)\left(l-k+\epsilon^{\tau}\right) n+\varphi^{\tau}(n)\right]}+N_{k} \\
=\sum_{t=1}^{2} \sum_{l=0}^{N-1} X_{l}^{t} H_{l}^{t} Q_{l-k}^{\tau}+N_{k}
\end{gathered}
$$

Where, $Y_{k}, X_{k}$ and $H_{k}$ are the frequency domain expression of $r(n), x(n), h(n) . N_{k}$ is the AWGN. $€$ is the normalized frequency offset and is given by $\Delta \mathrm{fT}$. $\mathrm{T}$ is the subcarrier symbol period. $\mathrm{Q}^{\tau}{ }_{\mathrm{L}}$ can be given by:

$Q_{L}^{\tau}=\frac{1}{N} \sum_{n=0}^{N-1} e^{j\left[\left(\frac{2 \pi}{n}\right)\left(L+\epsilon^{\tau}\right) n+\varphi^{\tau}(n)\right]}=$
$=\exp \left[j\left\{2 \pi\left(L+\in^{\tau}\right)+\varphi^{\tau}\right\}\left(\frac{1}{2}-\frac{1}{2 N}\right)\right] \frac{\sin \left[\frac{\left.2 \pi\left(L+\epsilon^{\tau}\right)+\varphi^{\tau}\right\}}{2}\right]}{N \cdot \sin \left[\frac{\left\{2 \pi\left(L+\epsilon^{\tau}\right)+\varphi^{\tau}\right\}}{2 N}\right]}$

At receiver, OFDM symbols are demodulated and the signal can be recovered from the relation of $\mathrm{Z}_{\mathrm{K}}=\mathrm{Y}^{1}{ }_{\mathrm{K}}+$ $\mathrm{Y}^{2}{ }_{\mathrm{K}} . \mathrm{Y}^{1}{ }_{\mathrm{K}}$ and $\mathrm{Y}_{\mathrm{K}}^{2}$ are the first antenna and the second antennas $\mathrm{K}^{\text {th }}$ subcarrier data. The innovative data can be detected through the detection process. For ease of system efficiency, performance and effectiveness analysis we assume that $Q \tau, t L=Q \tau L$.

\section{RESULTS AND DISCUSSION}

This section presents the simulation results to analyse the efficiency, performance and effectiveness of the proposed and effective cooperative beam forming algorithmic approach. The spatial diversity of the $8 \times 8$ system makes it the strongest contender for low or squat Bit Error Rate (BER). Signal to Noise ratio (SNR) v/s BER plot for (Quadrature Amplitude Modulation) QAM-16 modulations and fading channels have been shown. The FFT size of 128 is used. In the simulations, the research work makes use of the Rayleigh based fading MIMO and (Multiple Input and Single Output) MISO channels for the primary and secondary users. The enormous as well as diversified sub channels and cyclic and results are obtained for 4, 10 and 100 realizations of channels.

The efficiency, performance and effectiveness of the algorithmic approach and effective technique has been evaluated by computer simulations. The results indicated that the algorithmic approach, in addition to that to guarantee the required efficiency, performance and effectiveness of the PUs, increases spectrum usage efficiency by servicing the selected SUs. Here we have used 2x 1 and 2x2 MIMO- OFDM for transmitting and plot the result for BER vsEb/No. As we can see in the plot
$2 \times 2$ showing the best for BER as variation over the Eb/No. If we increase transmit and receive antenna in OFDM then the BER over the EB/No result will effective, improved and enhanced.

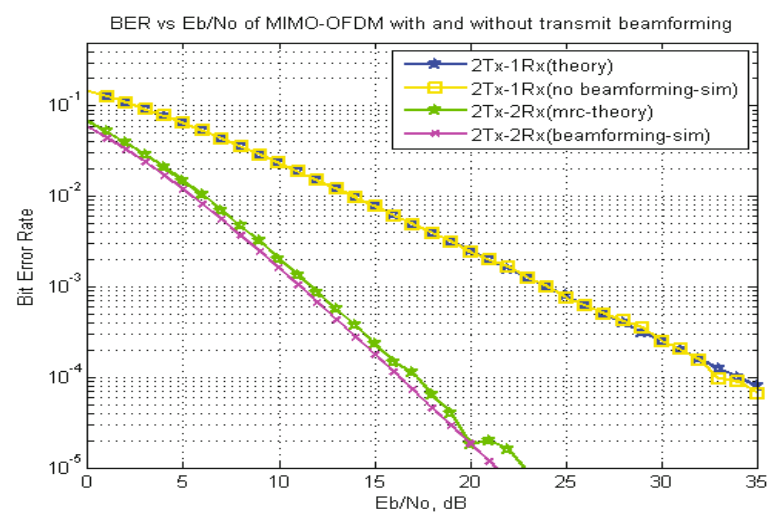

Figure 1. BER Vs Eb/No for MISO-OFDM and MIMOOFDM systems

In Figure 1, the implementation and simulation results depict the efficiency, performance and effectiveness of effective and better proposed cooperative algorithmic approach. In simulations, we make use of Raleigh fading based MISO as well as MIMO for the vital to discretionary customer. The enormous and developed sub-channels and cyclic prefix set with $16 \& 4$, autonomously. Meanwhile, simulation results are presented for 10 secondary users and 4, 100 primary users using MIMO technology and advancements. For SINR over SNR or abbreviated as dB), 10 secondary users and 100 PUs, showing the same simulation result, but the 4 PUs are differentiated and it after a certain SNR showing decreasing plot.

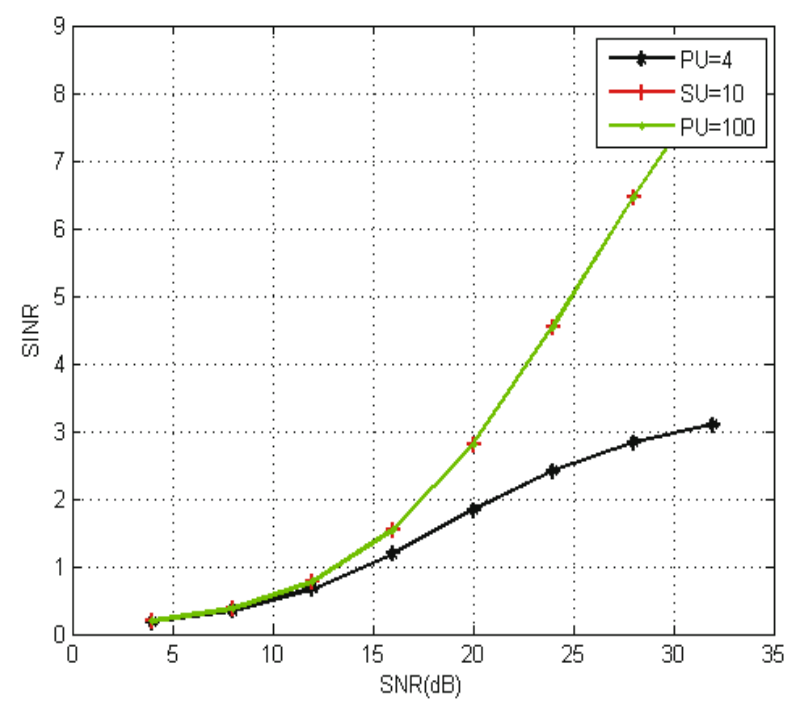

Figure 2. SINR related to primary and secondary user

Figure 2 shows the performance of PUs and SUs, from the fig, we can see the performance of the system is increased if the numbers of PUs user is more as compare to secondary users. Here, PUs $=100$ outperforms the PUs $=4$. 


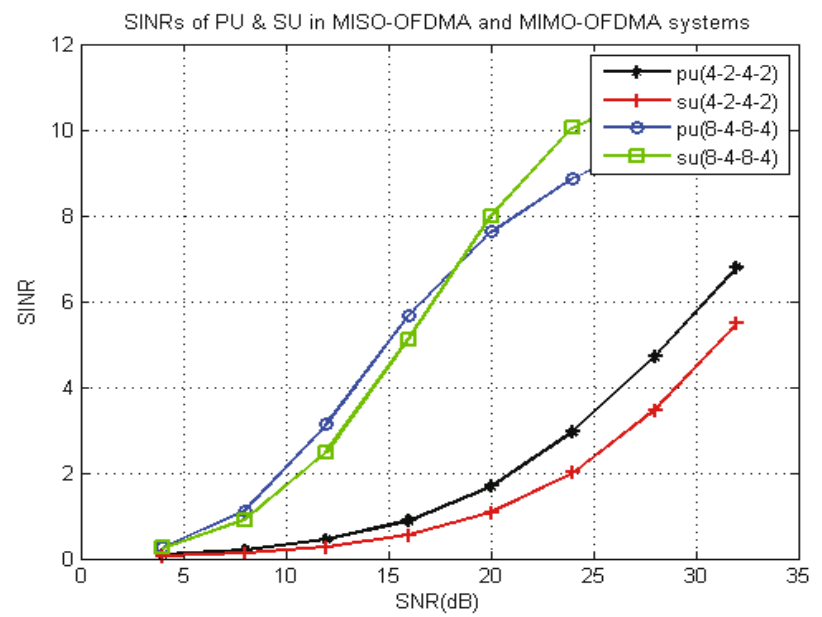

Figure 3. SINR of the PUs and SUs in MIMO-OFDM systems

Figure 3 depicts that there were 1 PUs and 2 SUs at the time enormous and diversified antennas are 4 for each of the transmitter and the receiver and the efficiency of spectrum is escalated a lot.

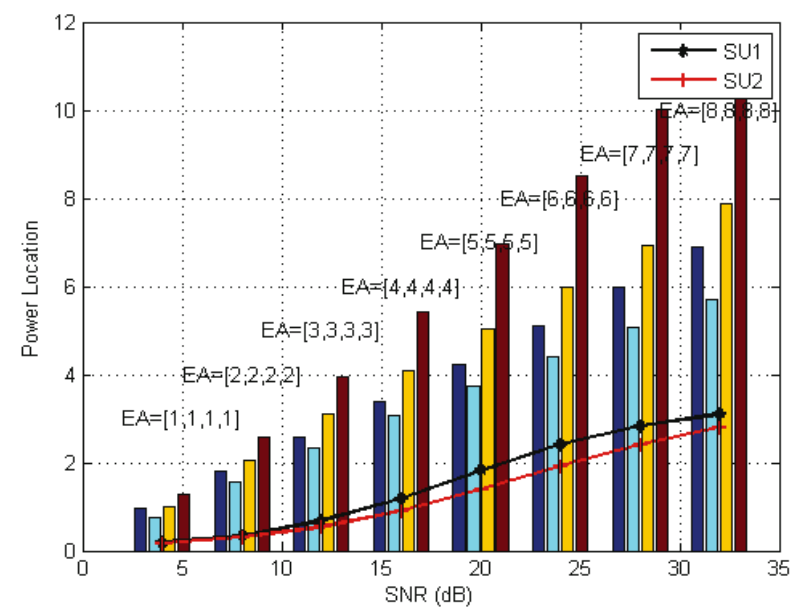

Figure 4. Power location over the SNR in MIMO system

Figure 4 shows that the symbol error rate for 16-QAM modulation. Where $\mathrm{N}$ is the No. of symbol and have multiple Es/No values, as there is phase ambiguity for phase $=0$ and $2 *$ pi, changing all phases reported as $2 *$ pi to 0 this is to enable comparison with the transmitted phase.

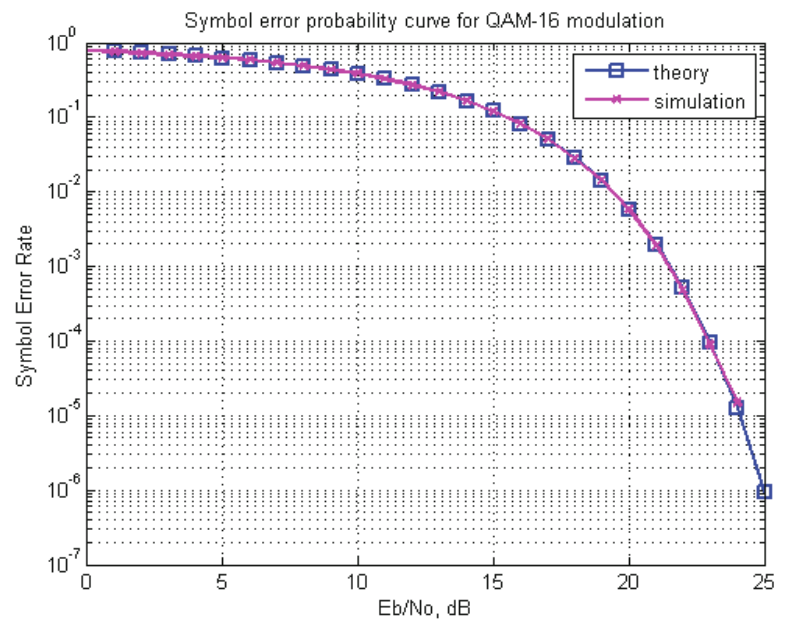

Figure 5. Symbol error rate vs Eb/No

Figure 5 shows the performance of Symbol error rate vs $\mathrm{Eb} /$ No where the performance of theory and simulated is matching for MIMO QAM-16 modulation schemes.

\section{CONCLUSION}

The work shows that MIMO-OFDM with $\mathrm{Cr}$ plays an important role in fifth generation mobile communication system (5G) to utilize the spectrum in an efficient manner. Beamforming technique reduces the interference, noise and also increase the capacity of the system. The proposed work concludes that with more numbers of antennas at transmitter and receiver will be the key technology for 5G. Severe as problem associated with this technology like security, internal- interference, power consumption, datarate can also be improved by using an efficient technology like Massive-MIMO, Forward Error Correcting Codes etc.

\section{DECLARATION}

We would like to state that this paper is original and only submitted to this journal.

\section{REFERENCES}

[1] Arun K, Manisha G., A Novel Modulation Technique for $5 \mathrm{G}$ Mobile Communication System. Communicated in American Journal Applied sciences 2015; 12(9): 601-605.

[2] Clerckx, Bruno, A practical cooperative multicell MIMO-OFDMA network based on rank coordination. IEEE Transactions on Wireless Transmissions 2014; 12(4): 1481-1491.

[3] Choi, Dongwook, Dongwoo L, Jae H., Resource allocation for COMP with multiuser MIMOOFDMA. IEEE Transactions on Vehicular Technology and advancements, 2011; 60(9): 46264632.

[4] Ghasemi, Amir, Elvino S. Sousa, Collaborative spectrum sensing for opportunistic access in fading environments. New Frontiers in Dynamic Spectrum 
Access Networks, DySPAN 2005, First IEEE International Symposium on. IEEE, 2005.

[5] Ghasemi, Amir, Elvino S. Sousa, Optimization of spectrum sensing for opportunistic spectrum access in cognitive radio networks, 4th IEEE Consumer Transmissions and Networking Conference, 2007.

[6] Arun K, Manisha G, Design, comparative study and analysis of CDMA for different modulation techniques, EGYPTIAN INFORMATIC JOURNAL 2015; 16(3): 351-365.

[7] Arun K, Manisha G., Design and Evaluation of BER for Ultra-Wide-Band System for Different Modulation Schemes, Emerging Research in Computing, Information, Communication and Applications, 97-108, Springer. India, BOOK: Electrical Engineering, ISBN: 978-81-322-2549-2. 2015.

[8] Md Islam, Y. Liang, A. Hoang, Joint power control and beam forming for cognitive radio network, IEEE TRANSACTIONS ON WIRELESS COMMUNICATIONS, 2008; 7(7): 2415-2419.

[9] W Jaafar, W Ajib, D Haccoun, Improving spectrum access using a beam-forming relay scheme for cognitive radio transmissions, IET COMMUNICATION, 2014, 8(7): doi:10.1049/ietcom1094-1103.

[10] G. Bansal, S. Toshniwal, A. Kumar, Cognitive Radio system with OFDM and Beam forming Technique for Data communication. $1^{\text {ST }}$ IEEE INTERNATIONAL CONFERENCE ON POWER ELECTRONICS. INTELLIGENT CONTROL AND ENERGYSYSTEMS, 2016, 1-3.

[11] X Lian, H Nikookar, Green Communications and Positioning by Integration of Adaptive and Distributed Beam-forming Technologies in Cognitive Radio Systems, IEEE, 978-1-4577-07872/11, 2011.

[12] Arun K, Manisha G. A Review on OFDM and PAPR Reduction Techniques, American Journal of Engineering and Applied Sciences (AJEAS) 2015; 8(2): 202-209.

[13] V. K. Dwivedi, G. Singh, An efficient BER analysis of OFDM systems with ICI conjugate cancellation method, PIERS Proceedings, Cambridge, USA, 2008.

[14] Arun K, Manisha G, Wireless Pers Commun (2017). doi:10.1007/s11277-017-4099-1. 